\title{
Effect of an intervention targeting inappropriate continued empirical parenteral vancomycin use: a quasi- experimental study in a region of high MRSA prevalence
}

Pyoeng Gyun Choe ${ }^{1,2+}$, Hei Lim Koo ${ }^{2 \dagger}$, Doran Yoon ${ }^{1}$, Ji Yun Bae ${ }^{1}$, Eunyoung Lee ${ }^{1}$, Joo-Hee Hwang ${ }^{1,3}$, Kyoung-Ho Song ${ }^{1}$, Wan Beom Park ${ }^{1,2}$, Ji Hwan Bang ${ }^{1}$, Eu Suk Kim¹', Hong Bin Kim', Sang Won Park', Myoung-don $\mathrm{Oh}^{1,2}$ and Nam Joong Kim ${ }^{1,2^{*}}$ (i)

\begin{abstract}
Background: Despite vancomycin use is a major risk factor for the emergence of vancomycin resistance, it is frequently inappropriately prescribed, especially as empirical treatment. We evaluated the effect of an antimicrobial stewardship intervention targeting for inappropriate continued empirical vancomycin use.

Methods: This was a quasi-experimental study comparing vancomycin use in a 6-month pre-intervention and 6month intervention period. If empirical vancomycin was continued for more than $96 \mathrm{~h}$ without documentation of beta-lactam-resistant gram-positive microorganisms, it was considered inappropriate continued empirical vancomycin use. The intervention consisted of the monitoring of appropriateness by a pharmacist and direct discussion with the prescribing physicians by infectious disease specialists when empirical vancomycin was continued inappropriately. An interrupted time series analysis was used to compare vancomycin use before and during the intervention.
\end{abstract}

Results: Following implementation of the intervention, overall vancomycin consumption decreased by $14.6 \%$, from 37.6 defined daily doses (DDDs)/1000 patient-days in the pre-intervention period to 32.1 DDDs/1000 patient-days in the intervention period $(P<0.001)$. The inappropriate consumption of vancomycin also declined from 8.0 DDDs/ 1000 patient-days to 5.8 DDDs/1000 patient-days $(P=0.009)$.

Conclusion: Interventions such as direct communication with prescribing physicians and infectious disease clinicians can help reduce the inappropriate continued use of vancomycin.

Keywords: Vancomycin, Inappropriate use, Antimicrobial stewardship, Intervention, Pharmacist, Infectious disease specialist

\footnotetext{
* Correspondence: molder@unitel.co.kr

This study was presented in part at the 4th ICPIC (International Conference on Prevention \& Infection Control), Geneva, Switzerland, 20th - 23rd June, 2017 (Abstract No. 016).

${ }^{\dagger}$ Equal contributors

'Department of Internal Medicine, Seoul National University College of Medicine, 103 Daehak-ro Jongno-gu, Seoul 03080, Republic of Korea

${ }^{2}$ Infection Control Office, Seoul National University Hospital, Seoul, Republic of Korea

Full list of author information is available at the end of the article
}

(c) The Author(s). 2018 Open Access This article is distributed under the terms of the Creative Commons Attribution 4.0 International License (http://creativecommons.org/licenses/by/4.0/), which permits unrestricted use, distribution, and reproduction in any medium, provided you give appropriate credit to the original author(s) and the source, provide a link to the Creative Commons license, and indicate if changes were made. The Creative Commons Public Domain Dedication waiver (http://creativecommons.org/publicdomain/zero/1.0/) applies to the data made available in this article, unless otherwise stated. 


\section{Background}

Vancomycin use is a major risk factor for acquisition of vancomycin-resistant Enterococci (VRE) [1], and infectious diseases caused by VRE are associated with increased mortality and length of hospital stay [2]. Also, the use of unnecessary vancomycin exposes patients to the risk of nephrotoxicity [3]. In 1995 the Centers for Disease Control and Prevention (CDC) established guideline for the appropriate use of vancomycin to reduce vancomycin resistance [4]. Since that time, many clinical guidelines recommended that empirically initiated vancomycin should be stopped, even in immunocompromised hosts, such as those with neutropenia, if there is no evidence of beta-lactam-resistant gram-positive infection [5, 6]. Despite these guidelines, 20\% to $70 \%$ of vancomycin use is inappropriate [7-11].

In our institution, over the last 12 years, about $60 \%$ of $S$. aureus infections were methicillin-resistant (MRSA), and this proportion has not changed significantly [12], whereas vancomycin consumption has increased more than twofold, from 18 defined daily doses (DDDs) per 1000 patient-days to 40.0 DDDs per 1000 patient-days [13]. Our previous analysis showed that a quarter of total vancomycin use represented inappropriately continued empirical use [14]. We have been executing an antibiotics stewardship program including an educational program, a computer-assisted antibiotic prescribing program, antimicrobial formulary restriction, and a prior-approval program. Our prior approval program is not very restrictive: physicians who are going to prescribe vancomycin have to describe the indication on a vancomycin order form which is modified from the indications for vancomycin use developed by the CDC [4]. Prescribing physicians are recommended, but not obliged to obtain prior approval by an infectious diseases specialist. In March 2015, the Antimicrobial Control Team of Seoul National University Hospital established an intervention to improve the appropriateness of empirical vancomycin use. The intervention consisted of monitoring of appropriateness by a pharmacist and direct discussion between the prescribing physicians and infectious disease specialists when empirical vancomycin was continued inappropriately beyond $96 \mathrm{~h}$. We report the details of the intervention program and its effects on empirical vancomycin use.

\section{Methods}

\section{Study design}

This quasi-experimental study was performed in Seoul National University Hospital, a 1778-bed, university-affiliated tertiary hospital in South Korea. The study was divided into two 6-month periods with respect to the intervention targeting inappropriate continued empirical vancomycin use, which began on March 16th 2015. We defined a 6-month pre-intervention period from July 14th 2014 to January 13th 2015, with the subsequent 2-month regarded as a washout period. The intervention period was then defined as the 6-month from the start of the intervention on March 16th 2015 to September 15th 2015. The institutional review board of Seoul National University Hospital reviewed the study protocol and provided study approval. It waived the requirement for written consent (IRB registration number 1407-043-593).

\section{The intervention}

During the intervention period, the pharmacist of the Antibiotic Control Team reviewed the medical records of patients for whom parenteral vancomycin was prescribed every day. If empirical vancomycin was continued beyond $96 \mathrm{~h}$ without documentation of beta-lactam-resistant grampositive microorganisms, the pharmacist informed an infectious disease specialist of the Antibiotic Control Team that empirical vancomycin was being prescribed inappropriately. The infectious disease clinician then met with, or phoned, the prescribing physician, and urged him/her to discontinue the vancomycin. Direct communication with the relevant physicians was intended to take place within $48 \mathrm{~h}$ of the time when infectious disease clinician was informed of the inappropriate continued empirical vancomycin use. The infectious disease clinician left a medical record of the recommendation to the prescribing physician and the pharmacist of the Antibiotic Control Team later confirmed that vancomycin had been discontinued.

\section{Data collection and definitions}

We retrospectively reviewed the medical records of patients who had been prescribed at least one dose of parenterally administered vancomycin during the study period. We included only patients who were at least 18 years of age.

The primary outcomes of the study were the total amount of vancomycin prescribed, the amount of inappropriate continued empirical vancomycin use, and the total amount of vancomycin prescribed that constituted inappropriate continued empirical use. Inappropriate continued empirical vancomycin was defined as in our previous report [14]. Briefly, we considered vancomycin prescribed before obtaining the culture results to be empirical. When empirically prescribed vancomycin treatment was continued beyond $96 \mathrm{~h}$ without documentation of beta-lactam-resistant gram-positive microorganisms in clinical specimens with significance, the continuation was considered inappropriate, and the amount used thereafter was considered inappropriately used. Vancomycin was calculated as defined daily doses (DDDs) per 1000 patient-days in accordance with World Health Organization recommendations [15]. Prescriptions for the same patient that were separated by 8 days or more were considered independent. 


\section{Statistical analysis}

Descriptive results for continuous variables were expressed as median values and interquartile ranges (IQRs). Clinical characteristics were compared using the chi-square test and the Matt-Whitney test for categorical and continuous variables, respectively. An interrupted time series analysis was used to compare the amount of vancomycin use before and after the intervention. Data analyses were performed using SAS software (version 9.4; SAS Institute, Cary, North Carolina).

\section{Results}

\section{Vancomycin consumption in the pre-intervention period}

During the pre-intervention period, a total of 1450 prescriptions of parenterally administered vancomycin were provided for 1249 patients, corresponding to 37 . 6 DDDs/1000 patient-days. Of the 1450 prescriptions, 212 (14.6\%) were for specific treatment of documented infections, 347 (23.9\%) were prophylactic, and 891 (61.5\%) were empirical. The amounts consumed for specific treatment, prophylaxis, and empirical treatment were $8.5 \mathrm{DDDs} / 1000$ patient-days (22.5\%), 5.2 DDDs/1000 patient-days (13.8\%), and 23.9 DDDs/ 1000 patient-days $(63.7 \%)$ respectively.

\section{Intervention activity}

During the intervention period, a total of 1457 prescriptions of parenterally administered vancomycin were given to 1244 patients. Of the 1457 prescriptions, 908 (62.3\%) were given empirically and 272 (18.7\%) were continued inappropriately beyond $96 \mathrm{~h}$ and became candidates for the intervention. An infectious disease specialist intervened about 223 (82.0\%) of these 272 prescriptions; 148 prescriptions (66.4\%, of 223 prescriptions) were discontinued within $24 \mathrm{~h}$ of the intervention, but 75 (33.6\%) were continued.

\section{Vancomycin consumption in the intervention period}

During the intervention period, overall vancomycin consumption decreased by $14.6 \%$, from 37.6 DDDs/ 1000 patient-days in the pre-intervention period to 32.1 DDDs/1000 patient-days in the intervention period $(P<0.001$, Fig. 1). In the latter period the amounts consumed for specific treatment, prophylaxis, and empirical treatment were $6.5 \mathrm{DDDs} / 1000$ patient-days (20. 2\%), 5.7 DDDs/1000 patient-days (17.8\%), and 19.9 DDDs/ 1000 patient-days (62.0\%) respectively. During the intervention period, the consumption for empirical use also was declined significantly $(P=0.005$, Table 1$)$. The incidence of MRSA bloodstream infection was 0.112 per 1000 patientdays in the pre-intervention period and 0.147 per 1000 patient-days in the intervention period, with no significant difference between the two periods $(P=0.272)$.

\section{Inappropriate continued empirical vancomycin use}

We identified 575 patients for whom empirical vancomycin was continued inappropriately during this study: 303 in the pre-intervention period and 272 in the intervention period. The proportion of patients with hematologic malignancies among the patients in whom empirical vancomycin was continued inappropriately was significantly higher during the intervention period, but the other baseline and clinical characteristics of the two sets of patients did not differ

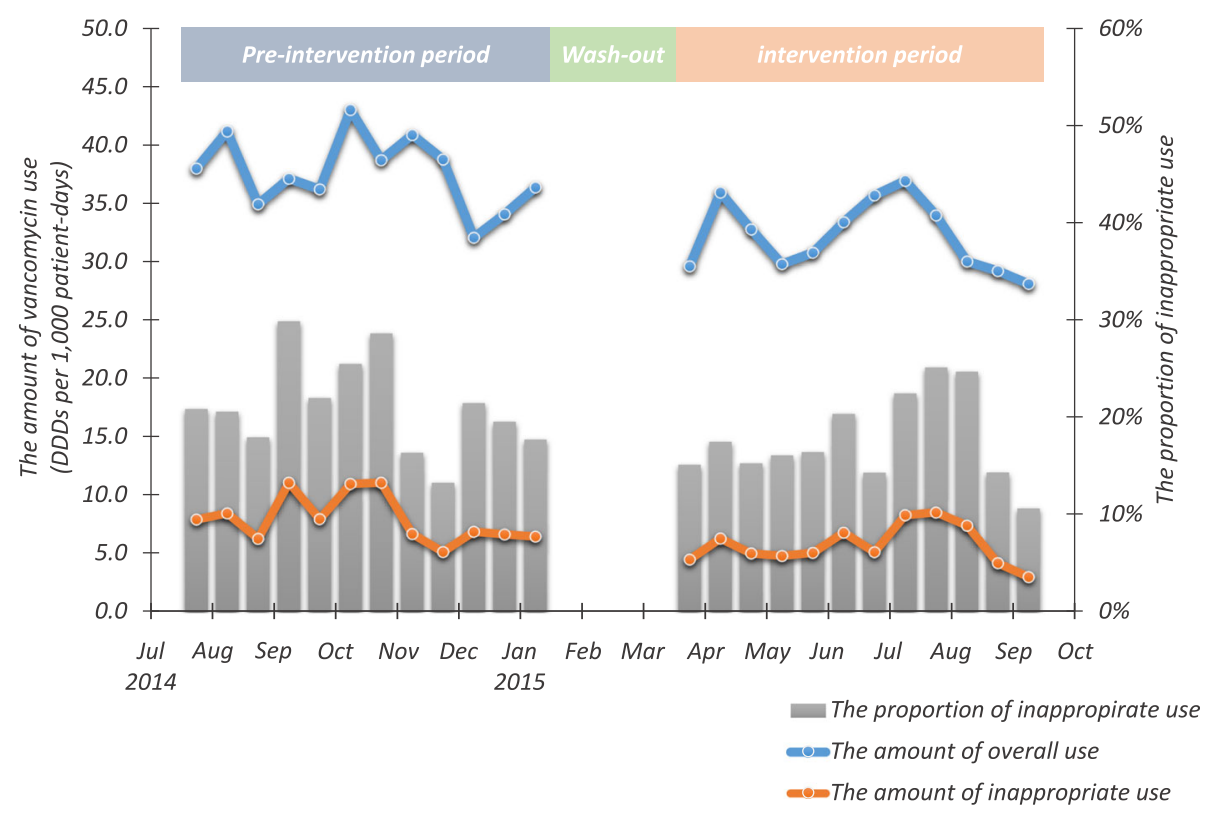

Fig. 1 Vancomycin consumption and appropriateness in the pre-intervention and intervention periods 
Table 1 The usage of vancomycin in the pre-intervention and intervention periods

\begin{tabular}{llll}
\hline & Pre-intervention period & Intervention period & $P$ value \\
\hline Total amount of vancomycin prescribed (DDDs/1000 patient-days) & 37.6 & 32.1 & $<0.001$ \\
Amount of empirical vancomycin (DDDs/1000 patient-days) & 23.9 & 19.9 & 0.005 \\
Amount of inappropriate continued empirical vancomycin (DDDs/1000 patient-days) & 8.0 & 5.8 & 0.009 \\
\hline
\end{tabular}

Abbreviations: $D D D$ defined daily dose

significantly (Table 2). In the pre-intervention period, the amount of inappropriate continued empirical vancomycin use was $8.0 \mathrm{DDDs} / 1000$ patient-days, representing $21.2 \%$ of the total parenterally administered vancomycin. In the intervention period, the inappropriate consumption of vancomycin declined to 5.8 DDDs $/ 1000$ patient-days $(P=0.009$, Fig. 1$)$. The proportion of vancomycin consumption amount that was continued inappropriately also decreased, but not significantly (21.2\% to $18.1 \%, P=0.087$ ).

Table 2 Characteristics of patients in whom empirical vancomycin was continued inappropriately during the pre-intervention and intervention periods ${ }^{a}$

\begin{tabular}{|c|c|c|c|}
\hline & Pre-intervention period & Intervention period & $P$ value \\
\hline No. of prescriptions & 303 & 272 & \\
\hline Male, $n(\%)$ & $181(59.7)$ & $163(59.9)$ & 0.963 \\
\hline Age, median years (IQR) & $60(48-71)$ & $61(49-72)$ & 0.528 \\
\hline \multicolumn{4}{|l|}{ Comorbid condition, $n$ (\%) } \\
\hline Diabetes mellitus & $68(22.4)$ & $53(19.5)$ & 0.385 \\
\hline Chronic liver disease & $28(9.2)$ & $27(9.9)$ & 0.780 \\
\hline Chronic lung disease & $10(3.3)$ & $1(0.4)$ & 0.010 \\
\hline Cerebrovascular disease & $10(3.3)$ & $11(4.0)$ & 0.635 \\
\hline Solid malignancy & $86(28.4)$ & $68(25.0)$ & 0.360 \\
\hline Hematological malignancy & $58(19.1)$ & $72(26.5)$ & 0.036 \\
\hline Connective tissue disease & $8(2.6)$ & $8(2.9)$ & 0.827 \\
\hline Azotemia & $46(15.2)$ & $46(16.9)$ & 0.572 \\
\hline Neutropenia & $61(20.1)$ & $63(23.2)$ & 0.378 \\
\hline Suspected site of infection, $n(\%)$ & & & 0.214 \\
\hline Pneumonia & $68(22.4)$ & $50(18.4)$ & \\
\hline Intraabdominal infection & $32(10.6)$ & $42(15.4)$ & \\
\hline CNS infection & $34(11.2)$ & $32(11.8)$ & \\
\hline Skin and soft tissue infection & $62(20.5)$ & $50(18.4)$ & \\
\hline Cardiovascular infection & $2(0.7)$ & $5(1.8)$ & \\
\hline Catheter-related infection & $25(8.3)$ & $16(5.9)$ & \\
\hline Bone and joint infection & $31(10.2)$ & $8(2.9)$ & \\
\hline Urinary tract infection & $5(1.7)$ & $3(1.1)$ & \\
\hline Other infection & $19(6.3)$ & $19(7.0)$ & \\
\hline Unknown & $25(8.3)$ & $47(17.3)$ & \\
\hline Admission department, $n$ (\%) & & & 0.303 \\
\hline Medial ward & $165(54.5)$ & $176(64.7)$ & \\
\hline Surgical ward & $86(28.4)$ & $46(16.9)$ & \\
\hline Medical ICU & $33(10.9)$ & $30(11.0)$ & \\
\hline Surgical ICU & $19(6.3)$ & $20(7.4)$ & \\
\hline 30-days mortality, n (\%) & $53(17.5)$ & 37 (13.6) & 0.200 \\
\hline
\end{tabular}

Abbreviations: CNS central nervous system, ICU intensive care unit

${ }^{a}$ When empirically prescribed vancomycin treatment was continued beyond $96 \mathrm{~h}$ without documentation of beta-lactam-resistant gram-positive microorganisms in clinical specimens with significance, the continuation was considered inappropriate 


\section{Discussion}

We have shown above that an intervention involving direct discussion between prescribing physicians and infectious disease clinicians increased the appropriateness of empirical vancomycin use. The intervention reduced the amount of inappropriate continued empirical vancomycin use from 8.0 to 5.8 DDDs/1000 patient-days, and the total amount of vancomycin prescribed represented by inappropriate continued empirical vancomycin use fell from $21.2 \%$ to $18.1 \%$.

There have been several investigations of the effects of various interventions aimed at improving vancomycin use, including pharmacists' interventions, automatic stop orders, antibiotic order forms, continuing education, computer-assisted antibiotic prescribing programs, antimicrobial formulary restrictions, and prior-approval programs. The effectiveness of pharmacists' interventions was demonstrated in a previous study [16]; the initial interventions were performed by pharmacists, and if inappropriate vancomycin use continued, a consultation with an infectious disease clinician was offered. As a result, the accord with guidelines for empiric use of vancomycin improved from $47 \%$ in the pre-intervention period to $73 \%$ during the intervention [16]. Another pharmacists' intervention consisting of contacting physicians and informing them of inappropriate vancomycin use significantly improved appropriate initiation of vancomycin [17]. These studies demonstrated that direct interventions of pharmacist and infectious disease clinicians with prescribers could be effective. An intervention consisting automatic $72 \mathrm{~h}$ stop orders also improved vancomycin prescribing [18]. However Bolon et al. reported that an antibiotic order form intervention did not improve or reduce vancomycin use [19], and the educational programs about vancomycin use failed to reduce inappropriate vancomycin prescribing in a prospective study [20].

An educational program, a computer-assisted antibiotic prescribing program, and a prior approval program have all been introduced in our institution to promote the prudent use of broad-spectrum antibiotics including vancomycin. Nevertheless, much of the vancomycin prescribed has been used inappropriately. In our opinion education and antibiotic order forms alone have little impact on the appropriateness of antibiotic use, and more intensive interventions such as direct discussions and compulsory stop orders would be more helpful for antimicrobial stewardship. Of course, such direct discussions with prescribing physician may not be long lasting, as it increases the demands on the time of infectious disease clinicians. Therefore, with this interventions, it is essential to establish local guidelines and policies for empirical prescribing. In Korea, to promote appropriate use of antibiotics, the Korea Centers for Disease Control and Prevention (KCDC) is carrying out a Policy Research Serving Project with related expert groups such as the Korean Society of Infectious Diseases, Korean Society for Chemotherapy since 2016 [21, 22].

Factors associated with the inappropriate use of vancomycin, such as critical clinical conditions, absence of documented causative organism, and suspected CNS infection, were identified in previous studies $[7,14]$. In this study, patients with hematologic malignancies accounted for a significantly higher proportion of the patients in whom empirical vancomycin was continued inappropriately during the intervention period than during the preintervention period. This suggests that physicians treating patients with hematologic malignancies tend to be less compliant with intervention instructions.

Our study has several limitations. First, we only focused on reducing the amount of inappropriate continued empirical vancomycin use. Further interventions focused on prophylaxis, specific treatments, and the initial choice of empirical vancomycin would also be helpful in reducing the total amount of vancomycin prescribed. Second, this was a non-randomized, pre/post-intervention study and the lack of random assignment weakens its significance. Third, we did not investigate why many prescribers continued with inappropriate empirical vancomycin use after discussion with infectious diseases clinicians.

\section{Conclusions}

Interventions such as direct communication with prescribing physicians and infectious disease clinicians can help reduce the inappropriate continued use of vancomycin.

\section{Abbreviations}

CDC: Centers for disease control and prevention; DDDs: Defined daily doses; IQRs: Interquartile ranges; MRSA: Methicillin-resistant Staphylococcus aureus; VRE: Vancomycin-resistant enterococci

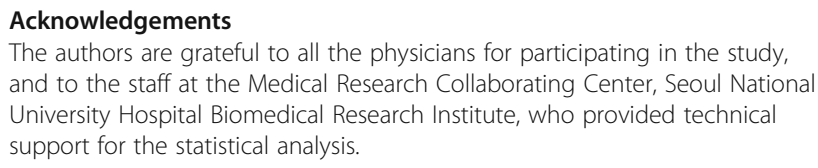
and to the staff at the Medical Research Collaborating Center, Seoul National University Hospital Biomedical Research Institute, who provided technical support for the statistical analysis.

\section{Funding}

This study was supported by a research grant from Seoul National University Hospital (Grant No. 2013-2425).

\section{Availability of data and materials}

The datasets used and analyzed during the current study are available from the corresponding author on reasonable request.

\section{Authors' contributions}

PGC and NJK designed the study. Acquisition of the data was performed by HLK, DY, JYB, EL and JHH. Analysis of the data was performed by PGC, KHS, WBP, JHB, ESK, HBK, SWB, MO and PGC wrote the final report. All authors interpreted data and contributed to the revision of the paper. All authors read and approved the final manuscript. 


\section{Ethics approval and consent to participate}

The institutional review board of Seoul National University Hospital reviewed the study protocol and provided study approval. It waived the requirement for written consent (IRB registration number 1407-043-593).

\section{Competing interests}

The authors declare that they have no competing interest.

\section{Publisher's Note}

Springer Nature remains neutral with regard to jurisdictional claims in published maps and institutional affiliations.

\section{Author details}

${ }^{1}$ Department of Internal Medicine, Seoul National University College of Medicine, 103 Daehak-ro Jongno-gu, Seoul 03080, Republic of Korea. ${ }^{2}$ Infection Control Office, Seoul National University Hospital, Seoul, Republic of Korea. ${ }^{3}$ Present address: Department of Internal Medicine, Chonbuk National University Medical School, Jeonju, Republic of Korea.

Received: 4 September 2017 Accepted: 3 April 2018

Published online: 16 April 2018

\section{References}

1. Carmeli Y, Samore MH, Huskins C. The association between antecedent vancomycin treatment and hospital-acquired vancomycin-resistant enterococci: a meta-analysis. Arch Intern Med. 1999;159(20):2461-8.

2. Salgado CD, Farr BM. Outcomes associated with vancomycin-resistant enterococci: a meta-analysis. Infect Control Hosp Epidemiol. 2003;24(9):690-8.

3. Sinha Ray A, Haikal A, Hammoud KA, Yu AS. Vancomycin and the risk of AKI: a systematic review and meta-analysis. Clin J Am Soc Nephrol. 2016;11(12): 2132-40.

4. The Hospital Infection Control Practice Advisory Committee. Recommendations for preventing the spread of vancomycin resistance: recommendations of the Hospital Infection Control Practices Advisory Committee (HICPAC). Am J Infect Control. 1995;23(2):87-94.

5. Lee DG, Kim SH, Kim SY, Kim CJ, Park WB, Song YG, et al. Evidence-based guidelines for empirical therapy of neutropenic fever in Korea. Korean J Intern Med. 2011;26(2):220-52.

6. Freifeld AG, Bow EJ, Sepkowitz KA, Boeckh MJ, Ito Jl, Mullen CA, et al. Clinical practice guideline for the use of antimicrobial agents in neutropenic patients with Cancer: 2010 update by the Infectious Diseases Society of America. Clin Infect Dis. 2011;52(4):e56-e93.

7. Junior MS, Correa L, Marra AR, Camargo LF, Pereira CA. Analysis of vancomycin use and associated risk factors in a university teaching hospital: a prospective cohort study. BMC Infect Dis. 2007;7:88

8. Melo DO, Ribeiro E. Vancomycin use in a Brazilian teaching hospital: comparison with the hospital infection Controlpractices advisory committee guidelines (HICPAC). Braz J Infect Dis. 2009;13(3):161-4.

9. Islahudin F, Ong HY. Appropriate vancomycin use in a Malaysian tertiary hospital based on current HICPAC recommendations. J Infect Dev Ctries 2014;8(10):1267-71.

10. Magill SS, Edwards JR, Beldavs ZG, Dumyati G, Janelle SJ, Kainer MA, et al. Prevalence of antimicrobial use in US acute care hospitals, may-September 2011. JAMA. 2014;312(14):1438-46.

11. Bollinger M, Hamilton M, Schroeder K, Link S, Nguyen J, Chu D, et al. Vancomycin use in a rural hospital: a 3-year retrospective study. Can J Rural Med. 2015;20(2):56-62.

12. Kim NH, Hwang JH, Song KH, Choe PG, Park WB, Kim ES, et al. Changes in antimicrobial susceptibility of blood isolates in a University Hospital in South Korea, 1998-2010. Infect Chemother. 2012;44(4):275-81.

13. Jun Kl, Koo HL, Kim MK, Kang CK, Kim MJ, Chun SH, et al. Trends in antibiotic use in a single university hospital. Korean J Nosocomial Infect Control. 2013;18(2):44-50

14. Kim NH, Koo HL, Choe PG, Cheon S, Kim M, Lee MJ, et al. Inappropriate continued empirical vancomycin use in a hospital with a high prevalence of methicillin-resistant Staphylococcus aureus. Antimicrob Agents Chemother. 2015;59(2):811-7.

15. Maxwell M, Heaney D, Howie JG, Noble S. General practice fundholding: observations on prescribing patterns and costs using the defined daily dose method. Br Med J. 1993;307(6913):1190-4.
16. Hamilton CD, Drew R, Janning SW, Latour JK, Hayward S. Excessive use of vancomycin: a successful intervention strategy at an academic medical center. Infect Control Hosp Epidemiol. 2000;21(1):42-5.

17. Chan S, Hossain J, Di Pentima MC. Implications and impact of prior authorization policy on vancomycin use at a tertiary pediatric teaching hospital. Pediatr Infect Dis J. 2015;34(5):506-8.

18. Guglielmo BJ, Dudas V, Maewal I, Young R, Hilts A, Villmann M, et al. Impact of a series of interventions in vancomycin prescribing on use and prevalence of vancomycin-resistant enterococci. Jt Comm J Qual Patient Saf. 2005;31(8):469-75.

19. Bolon MK, Arnold AD, Feldman HA, Goldmann DA, Wright SB. An antibiotic order form intervention does not improve or reduce vancomycin use. Pediatr Infect Dis J. 2005:24(12):1053-8.

20. Misan GM, Martin ED, Smith ER, Somogyi AA, Bartholomeusz RC, Bochner F. Drug utilization review in a teaching hospital: experience with vancomycin. Eur J Clin Pharmacol. 1990;39(5):457-61.

21. Yoon YK, Park CS, Kim JW, Hwang K, Lee SY, Kim TH, et al. Guidelines for the antibiotic use in adults with acute upper respiratory tract infections. Infect Chemother. 2017;49(4):326-52

22. Kwak YG, Choi SH, Kim T, Park SY, Seo SH, Kim MB, et al. Clinical guidelines for the antibiotic treatment for community-acquired skin and soft tissue infection. Infect Chemother. 2017:49(4):301-25.

\section{Ready to submit your research? Choose BMC and benefit from:}

- fast, convenient online submission

- thorough peer review by experienced researchers in your field

- rapid publication on acceptance

- support for research data, including large and complex data types

- gold Open Access which fosters wider collaboration and increased citations

- maximum visibility for your research: over $100 \mathrm{M}$ website views per year

At BMC, research is always in progress.

Learn more biomedcentral.com/submissions 